\title{
InteraChem: Virtual Reality Visualizer for Reactive Interactive Molecular Dynamics
}

Stefan Seritan, ${ }^{1,2}$ Yuanheng Wang, ${ }^{1,2}$ Jason E. Ford, ${ }^{1,2}$ Alessio Valentini, ${ }^{1,2}$ Tom Gold, ${ }^{1}$ and

Todd J. Martínez ${ }^{1,2, *}$

${ }^{1}$ Department of Chemistry and The PULSE Institute, Stanford University, Stanford, CA 94305

${ }^{2}$ SLAC National Accelerator Laboratory, 2575 Sand Hill Road, Menlo Park, CA 94025

\begin{abstract}
Interactive molecular dynamics in virtual reality (IMD-VR) simulations provide a digital molecular playground for students as an alternative or complement to traditional molecular modelling kits or 2D illustrations. Previous IMD-VR studies have used molecular mechanics to enable simulations of macromolecules such as proteins and nanostructures for the classroom setting with considerable success. Here, we present the INTERACHEM molecular visualizer, intended for reactive IMD-VR simulation using semiempirical and ab initio methods. INTERACHEM visualizes not only the molecular geometry, but also 1) isosurfaces such as molecular orbitals and electrostatic potentials, and 2) two-dimensional graphs of time-varying simulation quantities such as kinetic/potential energy, internal coordinates, and user-applied force. Additionally, INTERACHEM employs speech recognition to facilitate user interaction and introduces a novel "atom happiness" visualization using emojis to indicate the energetic feasibility of a particular bonding arrangement. We include a set of accompanying exercises that we have used to teach chemical reactivity in small molecular systems.
\end{abstract}




\section{Introduction}

Traditional molecular representations perform well for pedagogical purposes on small model systems but may struggle to accurately depict the full breadth of possible chemical reactions or the dynamic nature of molecules. For example, Kekule-Lewis structures and arrowpushing mechanisms are widely used to conceptualize organic chemistry reactions, but fail to emphasize the three-dimensional nature of the structures. Conversely, modeling kits provide a spatial representation of molecules, but physical limitations (such as the number and placement of holes in each atom) can make it difficult to describe some motions or chemical reactions and impossible to describe exotic species (for example, $\mathrm{CH}_{5}{ }^{+}$). Students can overcome these obstacles with training, but advances in modern technology suggest more direct approaches such as the use of virtual reality (VR). ${ }^{1-2}$ The rise of relatively affordable commodity VR headsets in the last few years has seen the development of a plethora of VR-enabled molecular visualizers. ${ }^{3-5}$ This has in turn fueled the growth of VR applications and activities for chemical education. ${ }^{6-11}$ Several studies have featured interactive molecular dynamics (IMD) simulations, where the user is immersed in a molecular movie with the ability to grab and pull molecules and observe how the system responds in real time. Haptic controllers allow control over translations and rotations and additionally have force-feedback capabilities, making them the traditional choice of peripheral for IMD. ${ }^{12-14}$ However, these controllers have a limited range of motion, are prohibitively costly for most classrooms, and do not have the same widespread familiarity and versatility as VR headsets. Therefore, IMD in VR (IMD-VR) simulations seem to be a promising alternative for increasing the accessibility and impact of these simulations throughout chemical education.

Smooth real-time simulations necessitate updates to the molecular geometry at least several times a second; therefore, evaluating the forces on the atoms must be done on the order of a tenth of second or faster. As a result, molecular mechanics (MM) are the most popular choice, enabling IMD to be applied to large macromolecules such as proteins and carbon nanotubes. ${ }^{15,6,4}$ However, MM force fields are not well-suited for studying chemical reactivity as bonds cannot be created or broken; therefore, semiempirical and ab initio methods are needed for reactive IMD simulations. The density functional tight binding ${ }^{16}$ (DFTB) method has been used to facilitate nanostructure construction and study chemical reactivity in systems with over 100 atoms. ${ }^{17-18}$ The use of consumer-grade graphical processing units (GPUs) enabled ab initio 
IMD (AI-IMD) for systems with dozens of atoms. ${ }^{19}$ Reactive IMD-VR simulations have been reported previously, ${ }^{4}$ but their potential impact for chemical education has not been fully explored.

Here, we present the INTERACHEM molecular visualizer and a set of exercises developed with reactive IMD-VR simulations for both high school and undergraduate chemistry students. The exercises cover a variety of topics, including molecular geometry and structure, molecular bonding and orbitals, conformational changes, and acid-base and organic reactivity. A large focus was placed on the visualization of quantum mechanical quantities (e.g. bond order, molecular orbitals, electron density) as these quantities are not well-described with traditional molecular modelling kits or MM-based IMD-VR simulations. The INTERACHEM visualizer and an accompanying worksheet that we have used in previous classroom demos are both available for free online..$^{20}$

\section{INTERACHEM - Virtual Reality Interactive Reactive Molecular Dynamics}

INTERACHEM is built with the UNITY game engine ${ }^{21}$ and can be deployed to a wide variety of peripherals. Our primary development was done with the Oculus Rift S, although the Oculus Rift and Quest (in tethered mode via Oculus Link) have also been used. ${ }^{22}$ Several stereoscopic (i.e. 3D) displays and projectors have been tested as well. The Rift S boasts "inside-out" tracking, meaning that no additional cameras or sensors are needed. This makes the INTERACHEM system fairly portable, which was useful as all three of our demonstrations required moving our simulation setup to a classroom. Additionally, haptic controllers such as the GEOMAGIC Touch ${ }^{23}$ are supported through the OPENHAPTICS UNITY plugin ${ }^{24-25}$ and can be used alongside a VR headset. This gives the advantages of both systems (i.e. 3D perception and force feedback), but the haptic controller's limited range of motion is still a major disadvantage compared to the freedom of the VR controllers.

A minimal control scheme was implemented with three functionalities: opening the menu (which pauses the simulation), selecting menu items or atoms, and panning/rotating the scene. The mappings of this scheme onto the VR handsets and haptic controllers are shown in Figure 1. Menu management (particularly drop-down menus for molecule and orbital selection) have been found to be challenging for users in VR. Table 1 lists a series of available voice commands using UNITY's built-in voice recognition module which has been added to circumvent some of these 
issues. Most notably, the "[load | get] molecule" command (where "molecule" is a specific molecular common name such as benzene or phenol) fetches a geometry via PubChem's Power User Gateway (PUG) REST API ${ }^{26}$ avoiding the onerous task of navigating a file explorer menu to start new simulations. The built-in recognition has some difficulty recognizing chemical names, and further work to resolve these issues and expand the capabilities of voice commands in INTERACHEM is ongoing.

Molecules are visualized using standard ball-and-stick representations, with the bond order dynamically updated by rounding the corresponding entries of the Mayer bond order matrix ${ }^{27}$ to the nearest half-integer. Atoms are highlighted when the controller hovers on them, and a yellow tether is displayed to indicate which atom is currently selected by the user. Isosurfaces for molecular orbitals, the full electron density, or the electrostatic potential can be displayed in realtime as shown in Figure 2. The user can request plots for kinetic and potential energy, temperature, or any internal coordinate (e.g. bond distance, angle, or torsion), as shown in Figure 3. These plots can be moved to any location in the VR scene, which we have found to be an excellent use for the additional space in the user's peripheral vision.

As an additional heuristic to aid and engage students, a "happiness" mode was added where happy and angry emojis were attached to each atom based on the local bonding environment, as demonstrated in Figure 4. The target number of bonds for first and second row elements is the difference between the number of valence electrons and the nearest full shell of electrons. For third row elements, hypervalency is possible and the set of allowed bonds ranges from the ideal number described above to the total number of valence electrons in increments of 2 (as each electron in a lone pair forms a new bond). We can define the difference between the target number of bonds and the current number of bonds as

$$
\Delta N_{i}=\left|N_{\text {ideal }}-\sum_{j \neq i} B O_{i j}\right|
$$

where $N_{\text {ideal }}$ is the closest ideal number of bonds and $B O_{i j}$ is the Mayer bond order between atoms $i$ and $j$. The "mood" of an atom is then determined by

$$
\operatorname{mood}_{i}= \begin{cases}\text { happy } & \Delta N_{i}<0.15 \\ \text { neutral } & 0.15<\Delta N_{i}<0.30 \\ \text { angry } & \Delta N_{i}>0.30\end{cases}
$$


The partial atomic charges (determined via Mulliken population analysis) and total molecular dipole are also shown in this mode, which can be activated by holding both triggers on the two VR controllers simultaneously.

The INTERACHEM visualizer is currently interfaced with two electronic structure packages. The xTB package ${ }^{28}$ is linked into the executable and provides the GFN2-xTB semiempirical method. ${ }^{29} \mathrm{Ab}$ initio methods such as Hartree-Fock (HF), density functional theory (DFT), configuration interaction singles (CIS), and complete active space configuration interaction (CASCI) are available from the TERACHEM electronic structure package via the recently developed TERACHEM Protocol Buffer (TCPB) interface. ${ }^{30-31}$ The TERACHEM server currently needs to be run on a separate machine from the INTERACHEM visualizer as TERACHEM does not currently support Windows, but further work is ongoing to resolve this technical issue. The majority of the exercises below can be performed with either backend; however, the GFN2-xTB method from the xTB package is used by default as it is faster and therefore provides a consistently smooth user experience. The details of these interfaces and benchmarking of the electronic structure methods for reactive IMD-VR purposes will be described in an upcoming publication.

The reactive IMD simulations use velocity Verlet integration, a Bussi-Parrinello Langevin thermostat, ${ }^{32}$ and a multiple timestep scheme incorporating the user force as in the AI-IMD work. ${ }^{19}$ We intentionally use a fast frictional relaxation time of $50 \mathrm{fs}$ in the thermostat (i.e., this is a "strong" thermostat) order to compensate for the large forces (on the molecular scale) that are often induced by user interaction. By promoting fast energy dissipation, the possibility of molecular rupture due to unintentionally large user forces is minimized. A spherical reflecting boundary ensures that atoms are not lost when molecules do rupture after being subjected to high forces. All simulations are carried out in the gas phase, although implementations for the generalized Born/surface area ${ }^{33-34}(\mathrm{~GB} / \mathrm{SA})$ and several conductor-like polarization models ${ }^{35-38}$ are available in XTB and TERACHEM, respectively, paving the way for solvent phase reactive IMD in the future. The user-applied force is implemented as a spring between the selected atom and the controller. The default spring constant is set to comfortably manipulate internal motions (e.g. angles, dihedrals), and the user can scale the spring constant by a multiplicative factor to break bonds or move more massive atoms. In addition to reactive IMD, one can also perform geometry optimization via an internal $\mathrm{BFGS}^{39-42}$ optimizer. Normal modes can be visualized 
from an optimized structure. Atoms can be frozen in space to run constrained dynamics or optimizations, or directly moved while the simulation is paused.

\section{Teaching Chemistry with INTERACHEM - Overview of Sample Exercises}

We have developed a series of exercises for reactive IMD-VR designed to be taught by one instructor with a single INTERACHEM-enabled station. Our demonstrations used a gaming laptop, the Oculus Rift S headset, and a few modelling kits. This portable solution allowed us to visit classrooms easily. Students take turns completing exercises while the remaining students observe the simulation on a second display or projector (usually already available in the classroom). The exercises begin with several basic molecular geometry tasks, such as attempting to distort tetrahedral methane, the umbrella flip motion in ammonia, or pseudorotations in trigonal bipyramidal complexes such as $\mathrm{PF}_{5}$. These serve as a general introduction to VR and IMD, and also provide a launching pad for instructor-led discussions of chemical bonding concepts like Valence Shell Electron Pair Repulsion (VSEPR) theory ${ }^{43}$ and the pitfalls of Kekule-Lewis structures and physical modelling kits. The next theme is reactivity and molecular orbitals, involving challenges such as comparing $\mathrm{C}-\mathrm{C}$ torsion for ethane vs. ethene, visualizing $\sigma$ and $\pi$ orbitals in ethene and benzene, and promoting the ring opening of cyclobutene (as showcased in Figure 5). Several exercises focus on benzene and cyclohexane, including a discussion of molecular planarity, aromaticity and the $\pi$ system in benzene (in which students attempt to throw a hydrogen atom through the ring), and converting between the chair and boat conformers of cyclohexane. Finally, chemical reactivity is first shown through proton transfers in several acidbase systems and then through standard organic reactions such as nucleophilic substitution and elimination reactions. Links for videos depicting each exercise can be found listed in Table 2 and a full discussion of the exercise goals, typical outcomes, and talking points can be found in the Supporting Information.

We first presented these exercises to 15 undergraduate students in a section of Chem 173 at Stanford University, the physical chemistry class introducing quantum mechanics. Together, the students completed the majority of the exercises in the 50-minute section and spent roughly equal time becoming comfortable with the VR setup as performing the exercises. The discussion was tailored to build on their chemical and mathematical background. For example, harmonic oscillators as models of vibration were included in the context of normal mode analysis and 
molecular mechanics. Additionally, wavefunction-related concepts such as the nodes and energy ordering of molecular orbitals and the electron density were highlighted.

We also demonstrated reactive IMD-VR to high school students on two occasions. A total of 46 high school students (primarily $11^{\text {th }}$ and $12^{\text {th }}$ graders) were split over four 50 -minute sessions as class C7382 at Stanford SPLASH Fall 2019. These sessions had two INTERACHEM stations and three instructors, giving small groups with five or six students per setup. Only half the exercises were run with a higher focus on molecular geometry (e.g. ammonia umbrella flip, cyclohexane conformers) and single vs. double bonds (e.g. ethane vs ethene torsion). The final challenge was setup as competition between the two teams with the goal to perform an $\mathrm{S}_{\mathrm{N}} 2$ reaction in a hydroxide and methyl fluoride system. Most groups were able to discover the mechanism in reactive IMD-VR after three or four tries despite having no organic chemistry instruction. Finally, an extended 1.5-hour workshop was run for 17 high school students from $9^{\text {th }}$ to $12^{\text {th }}$ grade with one INTERACHEM setup. Four of these students evidently had previous exposure to VR headsets and were immediately comfortable in the simulation. In general, the high school students quickly acclimated to VR environment but spent longer completing each exercise compared to the undergraduates; from our experiences, high school students need at least two hours to complete all exercises that the undergraduates completed in 50 minutes.

We found the energy plots and molecular modeling kits to be valuable complementary tools for the instructor and external students. The instructor can point out energy barriers and relative stability of the conformers in real time, while the modeling kits provided reference structures to anchor students to their previous lessons. For students who completed exercises quickly, the challenges were further "gamified" by adding additional constraints, such as only allowing them to touch certain atoms or reducing the user-applied force. In the future, these concepts could be quantified to provide a numerical score for each challenge, where the lowest score represents the discovery of the minimum energy mechanism with the smallest amount of external force. Conversely, students also greatly enjoyed using high forces to easily pull apart molecules. In addition to increasing student interest, relevant discussion can be generated by focusing on the recombination products in these scenarios. 


\section{Conclusion}

IMD-VR simulations provide an excellent complement to traditional modeling kits by allowing students to visualize and interact with molecules in a $3 \mathrm{D}$ digital playground. Previous work has used molecular mechanics in IMD-VR simulations to expose students to proteins and nanostructures with great success. The use of semiempirical and $a b$ initio molecular dynamics enables reactive IMD-VR simulations on system sizes typically taught in general and organic chemistry, providing an alternative way for students to study chemical reactivity without resorting to heuristics or rote memorization from textbooks. The IMD simulations may even occasionally appear to disagree with the textbooks, leading into discussions on advanced topics such as solvation effects to stabilize charged species in the nucleophilic substitution exercises.

INTERACHEM is a UNITY-based visualizer with interfaces to the xTB and TERACHEM quantum chemistry programs designed for reactive IMD-VR simulations. As a result, INTERACHEM focuses on visualization of wavefunction information in the form of adaptive bond order and molecular orbital or electron density isosurfaces that are not available in MM-based IMD-VR. A set of exercises for reactive IMD-VR have been developed and demonstrated for almost 80 students across both high school and undergraduate levels. In general, both groups of students responded well to the VR simulations, although high school students took longer to complete the exercises. However, this does not mean the high school students had a less successful experience; on the contrary, the high school students had animated discussions and provided creative (even if unintended) solutions.

Although not quantified here, the concept of "gamification" for reactive IMD-VR simulations in the form of minimizing the user-applied force or constraining which atoms can be manipulation emerged naturally during our demonstrations. Gamification has seen significant success for both increasing participation and providing creative solutions to scientific problems such as protein folding. ${ }^{44-45}$ Further study is warranted on whether the gamification of reactive IMD-VR simulations leads to increased student participation or greater completion of learning objectives.

\section{Supporting Information}

Document including in-depth discussions of each sample exercises, as well as the worksheet with sample exercises discussed in the text. 


\section{Acknowledgements}

This work was supported by the Center for Quantum Molecular Design (CQMD) through the National Science Foundation (CHE-1740645). S.S. and J.E.F. acknowledge support from NSF Graduate Research Fellowships. T.G. was supported by a Summer Undergraduate Research Fellowship. 

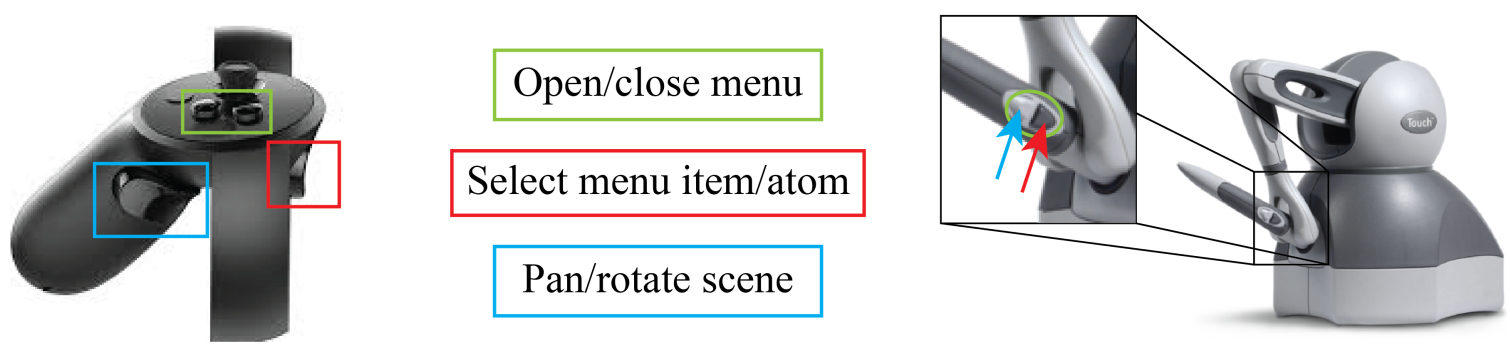

Figure 1. Basic control scheme for both VR (left) and haptic (right) controllers. Voice control can be accessed via the $\mathrm{X}$ button on the Oculus Touch controllers or the S key on the keyboard. 

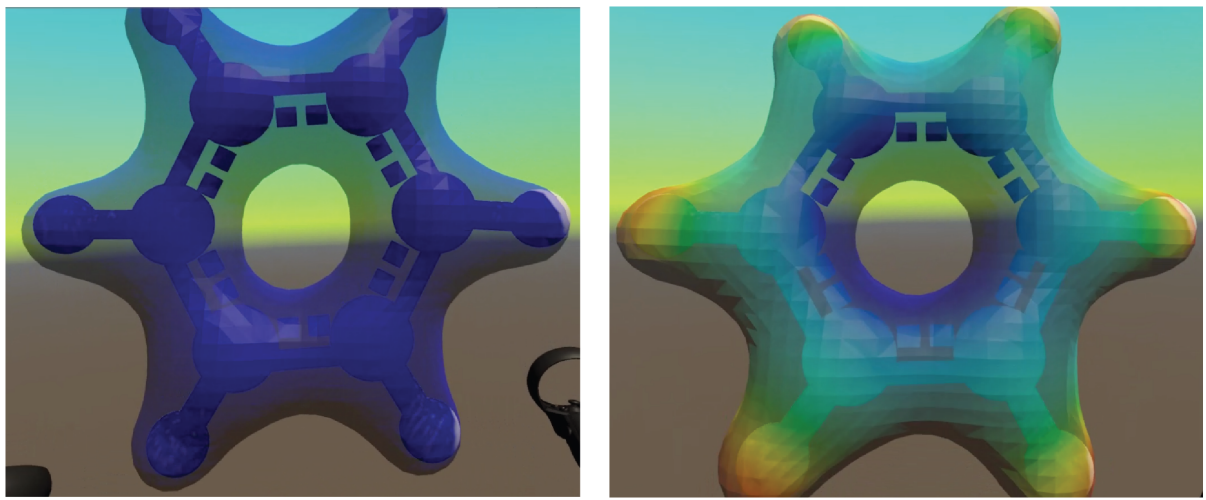

Figure 2. Electron density (left) and electrostatic potential (right) isosurfaces for benzene. The electrostatic potential uses the density isosurface but colors according to charge (blue for negative, red for positive). 

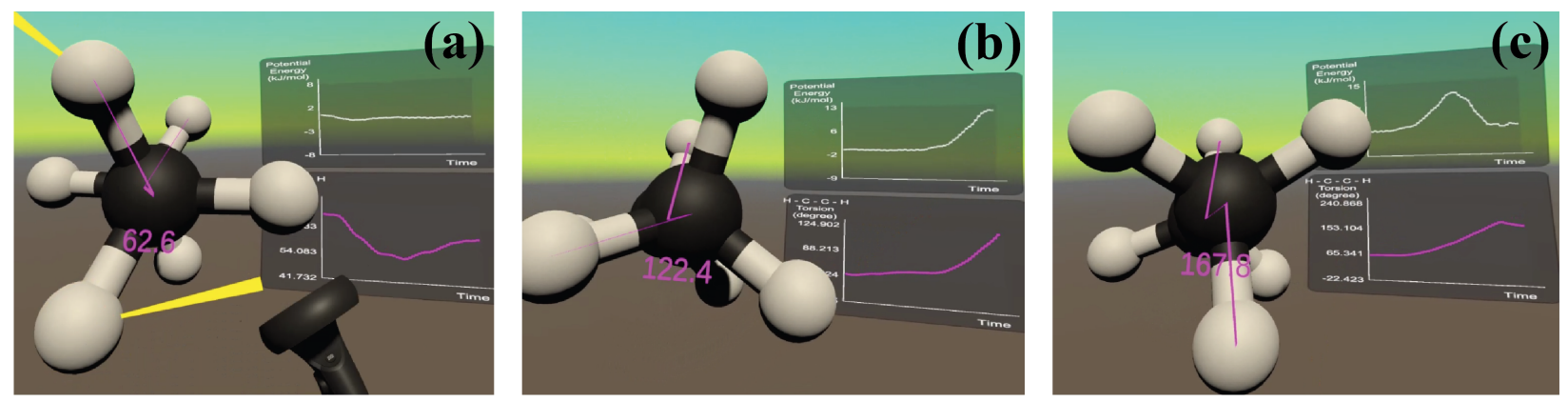

Figure 3. Ethane torsion showcasing potential energy in upper white plot and $\mathrm{H}-\mathrm{C}-\mathrm{C}-\mathrm{H}$ torsion in lower purple plot for (a) staggered conformation at $60^{\circ}$, (b) eclipsed at $120^{\circ}$, and finally (c) staggered again at $180^{\circ}$. Note the plot y-axes change automatically rescale from (a) to (b) to fit the new range of values. 

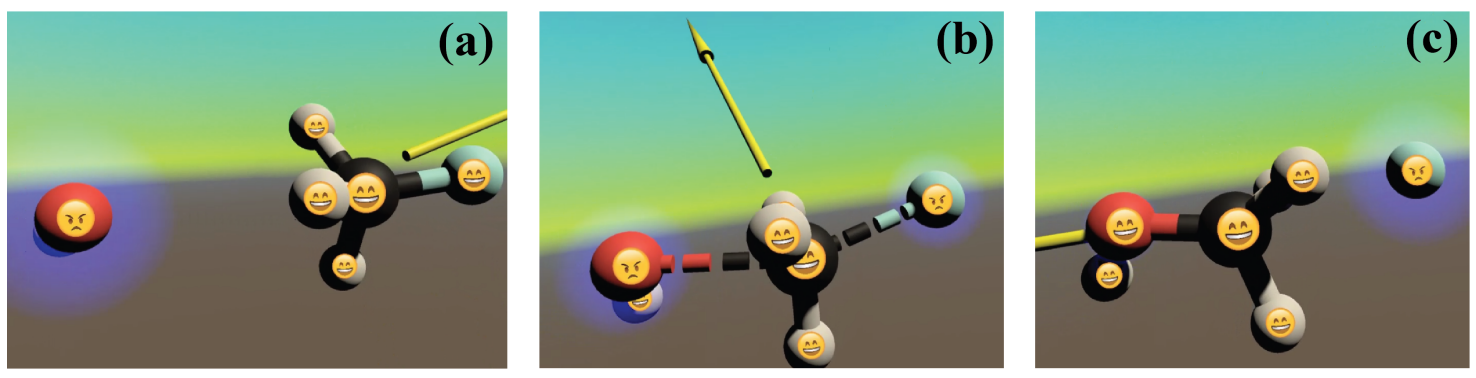

Figure 4. Atomic charge and emojis representing the local environment via "happiness". The yellow arrow is the molecular dipole, the blue aura represents negative charge, and an atom is labeled as happy/angry based on a bond saturation heuristic. This $\mathrm{S}_{\mathrm{N}} 2$ reaction starts with (a) the negatively charged oxygen as unhappy, (b) both the nucleophile and leaving group are unhappy at the transition state as negative charge is transferred, and (c) the fluoride ion is unsaturated upon reaction completion. 

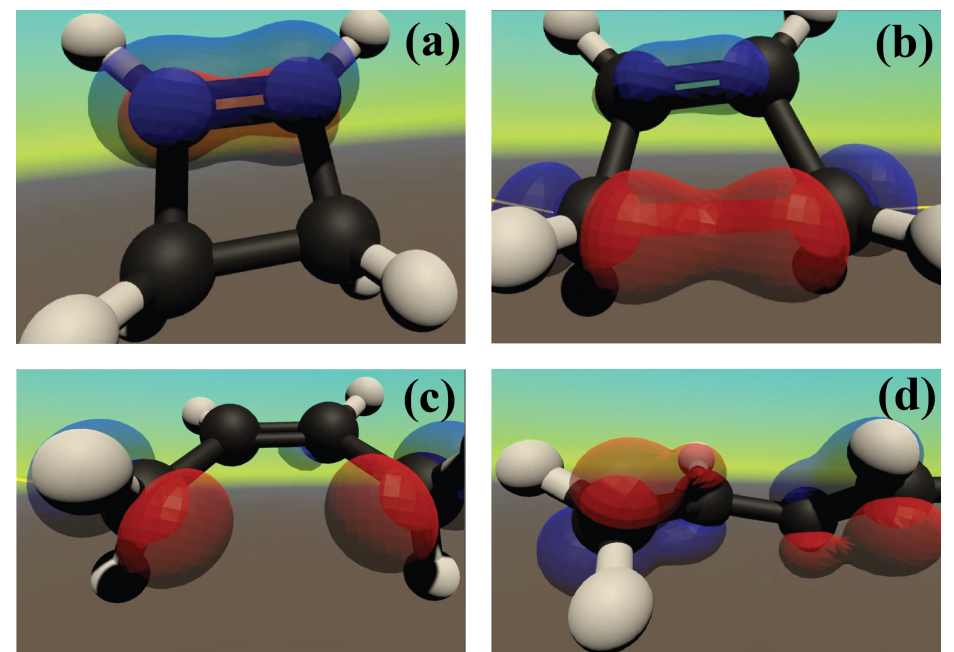

Figure 5. Ring opening of cyclobutene to butadiene displaying the highest occupied molecular orbital (HOMO), which starts as (a) a pi bond, (b) migrates to the breaking sigma bond as force is applied, (c) splits into individual $p$ orbitals near the transition state, and (d) forms the new pi system of butadiene. Orbitals are prone to fluctuating over the course of the simulation and these snapshots are curated to demonstrate general trends in the evolution of the HOMO. 
Table 1. Available voice commands for InteraChem. Square braces separated by a pipe indicate interchangeable keywords, trailing question marks indicate optional keywords, and angle braces denote input variables.

\begin{tabular}{|l|l|}
\hline Phrase & Effect \\
\hline help & List all recognizable phrases \\
\hline [pause $\mid$ stop] & Pause the simulation \\
\hline [play $\mid$ resume] & Resume the simulation \\
\hline Temperature $<$ T $>$ & Set the temperature to $<$ T $>$ Kelvin \\
\hline $\begin{array}{l}\text { [multiply } \mid \text { increase } \mid \text { scale] } \\
\text { force }[\text { by]? }<\text { factor }>\end{array}$ & Set the user-applied force multiplier to $<$ factor $>$ \\
\hline $\begin{array}{l}{[\text { load } \mid \text { get] molecule }} \\
<\text { name }>\end{array}$ & Fetch a molecular geometry via PubChem PUG REST API \\
\hline [optimize $\mid$ minimize] & Run geometry optimization on the current structure \\
\hline $\begin{array}{l}\text { normal mode }<\text { mode }> \\
\text { select }[\text { bond } \mid \text { bond length] }\end{array}$ & $\begin{array}{l}\text { Visualize mode }<\text { mode }>\text { (listed in descending order by frequency, } \\
\text { only available after minimization) }\end{array}$ \\
\hline select [angle $\mid$ bond angle] & Begin bond angle selection for a new plot \\
\hline $\begin{array}{l}\text { select }[\text { dihedral } \mid \text { dihedral } \\
\text { angle } \mid \text { torsion angle] }\end{array}$ & Begin dihedral angle selection for a new plot \\
\hline $\begin{array}{l}\text { orbital }<\text { n }> \\
\text { [highest occupied } \mid \text { lowest } \\
\text { unoccupied] molecular } \\
\text { orbital }\end{array}$ & $\begin{array}{l}\text { Visualize canonical orbital }<\text { n }>\text { isosurface (listed in ascending } \\
\text { energy order) }\end{array}$ \\
\hline $\begin{array}{l}\text { electron density } \\
\text { electrostatic potential } \\
\text { [orbital } \mid \text { vensity] isovalue }>\end{array}$ & Set the isovalue of orbital or electron density isosurfaces \\
\hline
\end{tabular}


Table 2. Video demonstrations of several IMD-VR exercises

\begin{tabular}{|c|c|c|}
\hline Video & Exercise & URL \\
\hline 1 & Distorting tetrahedral methane & https://youtu.be/DJVWA8hyiLU \\
\hline 2 & Umbrella flip in ammonia & https://youtu.be/vHSqrbgNxuI \\
\hline 3 & Pseudorotations of $\mathrm{PF}_{5}$ and $\mathrm{PH}_{3} \mathrm{~F}_{2}$ & https://youtu.be/xKiVzd3kifc \\
\hline 4 & Ethane $\mathrm{C}-\mathrm{C}$ torsion & https://youtu.be/3UpAGv0GDhU \\
\hline 5 & Ethene $\mathrm{C}=\mathrm{C}$ torsion & https://youtu.be/VDNtnQqWpEE \\
\hline 6 & Ring opening of cyclobutene & https://youtu.be/WtIQXeA7PDY \\
\hline 7 & Hydrogen atom through benzene ring & https://youtu.be/Qn0v3rTVFRA \\
\hline 8 & Cyclobutane chair/boat conformer flip & https://youtu.be/b5H-Dn261Z0 \\
\hline 9 & Proton transfer in carbonic acid + water & https://youtu.be/E1B6gkPECKA \\
\hline 10 & $\mathrm{~S}_{\mathrm{N}} 2$ of methyl fluoride + hydroxide & https://youtu.be/iVll4L8yWHY \\
\hline 11 & E1 of t-butyl fluoride + hydroxide & https://youtu.be/evF5GoAOkHU \\
\hline 12 & High force decomposition & https://youtu.be/Hq6JD33OpBo \\
\hline
\end{tabular}




\section{References}

1. Limniou, M.; Roberts, D.; Papdopoulus, N., Full immersive virtual environment CAVETM in chemistry education. Comput. Educ. 2008, 51, 584-593.

2. Won, M.; Mocerino, M.; Tang, K.-S.; Treagust, D. F.; Tasker, R., Interactive Immersive Virtual Reailty to Enhance Students' Visualization of Complex Molecules. In Research and Practice in Chemistry Education, Schultz, M.; Schmid, S.; Lawrie, G., Eds. Springer: Singapore, 2019.

3. Norrby, M.; Grebner, C.; Eriksson, J.; Boströ, J., Molecular Rift: Virtual Reality for Drug Designers. J. Chem. Inf. Model. 2015, 55, 2475-2484.

4. O'Connor, M. B.; Bennie, S. J.; Deeks, H. M.; Jamieson-Binnie, A.; Jones, A. J.; Shannon, R. J.; Walters, R.; Mitchell, T. J.; Mulholland, A. J.; Glowacki, D. R., Interactive molecular dynamics in virtual reality from quantum chemistry to drug binding: An open-source multiperson framework. J. Chem. Phys. 2019, 150, 220901.

5. Nanome Inc. Creating powerful, collaborative and scientific VR tools | Nanome. https://nanome.ai/nanome (accessed April 23, 2020).

6. Bennie, S. J.; Ranaghan, K. E.; Deeks, H. M.; Goldsmith, H. E.; O’Connor, M. B.; Mulholland, A. J.; Glowacki, D. R., Teaching Enzyme Catalysis Using Interactive Molecular Dynamics in Virtual Reality. J. Chem. Ed. 2019, 96, 2488-2496.

7. Bibic, L.; Druskis, J.; Walpole, S.; Angulo, J.; Stokes, L., Bug Off Pain: An Educational Virtual Reality Game on Spider Venoms and Chronic Pain for Public Engagement. J. Chem. Ed. 2019, 96, 1486-1490.

8. Ferrell, J. B.; Campbell, J. P.; McCarthy, D. R.; McKay, K. T.; Hensinger, M.; Srinivasan, R.; Zhao, X.; Wurthmann, A.; Li, J.; Schneebeli, S. T., Chemical Exploration with Virtual Reality in Organic Teaching Laboratories. J. Chem. Ed. 2019, 96, 1961-1966.

9. Fung, F. M.; Choo, W. Y.; Ardisara, A.; Zimmermann, C. D.; Watts, S.; Koscielniak, T.; Blanc, E.; Coumoul, X.; Dumke, R., Applying a Virtual Reality Platform in Environmental Chemistry Education To Conduct a Field Trip to an Overseas Site. J. Chem. Ed. 2019, 96, 382386.

10. Dunnagan, C. L.; Dannenberg, D. A.; Cuales, M. P.; Earnest, A. D.; Gurnsey, R. M.; Gallardo-Williams, M. T., Production and Evaluation of a Realistic Immersive Virtual Reality Organic Chemistry Laboratory Experience: Infrared Spectroscopy. J. Chem. Ed. 2020, 97, 258262.

11. Martino, M.; Salvadori, A.; Lazzari, F.; Paoloni, L.; Nandi, S.; Mancini, G.; Barone, V.; Rampino, S., Chemical promenades: Exploring potential - energy surfaces with immersive virtual reality. J. Comput. Chem. 2020, 41, 1310-1323.

12. Brooks, F. P.; Ouh-Young, M.; Batter, J. J.; Kilpatrick, P. J., Project GROPEHaptic displays for scientific visualization. SIGGRAPH Comput. Graph. 1990, 24, 177-185.

13. Stone, J. E.; Gullingsrud, J.; Schulten, K. In A System for Interactive Molecular Dynamics Simulation, Proceedings of the 2001 Symposium on Interactive 3D Graphics, ACM: 2001; pp 191-194.

14. Magnanelli, E.; Brero, G.; Garnier, R. V. E.; Mazzoletti, G.; Rizzi, A. M.; Comai, S., HaptiChem: Haptic and Visual Support in Interactions with the Microscopic World. In International Conference on Learning and Collaboration Technologies, Zaphiris, P.; Ioannou, A., Eds. Springer: 2014; Vol. 8524.

15. Croll, T. I., ISOLDE: a physically realistic environment for model building into lowresolution electron-density maps. Acta Cryst. D 2018, 74, 519-530. 
16. Elstner, M.; Porezag, D.; Jungnickel, G.; Elsner, J.; Haugk, M.; Frauenheim, T.; Suhai, S.; Seifert, G., Self-consistent-charge density-functional tight-binding method for simulations of complex materials properties. Phys. Rev. B 1998, 58, 7260-7268.

17. Haag, M. P.; Reiher, M., Studying chemical reactivity in a virtual environment. Faraday Discuss. 2014, 169, 89-118.

18. Haag, M. P.; Vaucher, A. C.; Bosson, M.; Redon, S.; Reiher, M., Interactive Chemical Reactivity Exploration. ChemPhysChem 2014, 15, 3301-3319.

19. Luehr, N.; Jin, A. G. B.; Martínez, T. J., Ab Initio Interactive Molecular Dynamics on Graphical Processing Units (GPUs). J. Chem. Theory Comput. 2015, 11, 4536-4544.

20. Group, T. M. Software | The Martinez Group. https://mtzweb.stanford.edu/software (accessed July 6, 2020).

21. Unity Technologies Unity Real-Time Development Platform | 3D, 2D VR \& AR Visualizations. https://unity.com/.

22. Facebook Technologies LLC Oculus | VR Headsets \& Equipment. https://www.oculus.com/ (accessed July 7, 2020).

23. 3D Systems, I. Touch Haptic Device. https://www.3dsystems.com/haptics-devices/touch (accessed July 2, 2020).

24. 3D Systems, I. OpenHaptics Developer Software. https://www.3dsystems.com/hapticsdevices/openhaptics (accessed July 2, 2020).

25. Unity 3D Systems Openhaptics Unity Plugin | Integration | Unity Asset Store. https://assetstore.unity.com/packages/tools/integration/3d-systems-openhaptics-unity-plugin134024 (accessed July 2, 2020).

26. PubChem PUG REST. https://pubchemdocs.ncbi.nlm.nih.gov/pug-rest (accessed July 8, 2020).

27. Mayer, I., Charge, Bond Order and Valence in the Ab Initio SCF Theory. Chem. Phys. Lett. 1983, 97, 270-274.

28. Ehlert, S.; Spicher, S.; Lehtola, S.; Unsleber, J.; Pracht, P.; Seibert, J.; Musil, F.; Caldeweyher, E.; Bannwarth, C.; Koopman, J.; Grimme, S. xtb: Semiempirical Extended TightBinding Program Package, grimme-lab: GitHub, 2020.

29. Bannwarth, C.; Ehlert, S.; Grimme, S., GFN2-xTB - An Accurate and Broadly Parametrized Self-Consistent Tight-Binding Quantum Chemical Method with Multipole Electrostatics and Density-Dependent Dispersion Contributions. J. Chem. Theory Comput. 2019, 15, 1652-1671.

30. Seritan, S.; Bannwarth, C.; Fales, B. S.; Hohenstein, E. G.; Isborn, C. M.; Kokkila Schumacher, S. I. L.; Li, X.; Liu, F.; Luehr, N.; Snyder, J. W.; Song, C.; Titov, A. V.; Ufimtsev, I. S.; Wang, L. P.; Martínez, T. J., TeraChem: A graphical processing unit-accelerated electronic structure package for large-scale ab initio molecular dynamics. WIREs Comput. Mol. Sci. 2020, e1494.

31. Seritan, S.; Bannwarth, C.; Fales, B. S.; Hohenstein, E. G.; Kokkila Schumacher, S. I. L.; Luehr, N.; Snyder, J. W.; Song, C.; Titov, A. V.; Ufimtsev, I. S.; Martínez, T. J., TeraChem: Accelerating electronic structure and ab initio molecular dynamics with graphical processing units. J. Chem. Phys. 2020, 152, 224110.

32. Bussi, G.; Parrinello, M., Accurate sampling using Langevin dynamics. Phys. Rev. E 2007, 75, 056707.

33. Qui, D.; Shenkin, P. S.; Hollinger, F. P.; Still, W. C., The GB/SA Continuum Model for Solvation. A Fast Analytical Method for the Calculation of Approximate Born Radii. J. Phys. Chem. A 1997, 101, 3005-3014. 
34. Reddy, M. R.; Erion, M. D.; Agarwal, A.; Viswanadhan, W. N.; McDonald, D. Q.; Still, W. C., Solvation free energies calculated using the GB/SA model: Sensitivity of results on charge sets, protocols, and force fields. J. Comput. Chem. 1998, 19, 769-780.

35. Klamt, A.; Schüürmann, G., COSMO: a new approach to dielectric screening in solvents with explicit expressions for the screening energy and its gradient. J. Chem. Soc., Perkin Trans. 2 1993, 799-805.

36. Truong, T. N.; Stefanovich, E. V., A new method for incorporating solvent effect into the classical, ab initio molecular orbital and density functional theory frameworks for arbitrary shape cavity. Chem. Phys. Lett. 1995, 240, 253-260.

37. Barone, V.; Cossi, M., Quantum Calculation of Molecular Energies and Energy Gradients in Solution by a Conductor Solvent Model. J. Phys. Chem. A 1998, 102, 1995-2001.

38. Liu, F.; Luehr, N.; Kulik, H. J.; Martínez, T. J., Quantum Chemistry for Solvated Molecules on Graphical Processing Units Using Polarizable Continuum Models. J. Chem. Theory Comput. 2015, 11, 3131-3144.

39. Broyden, C. G., The Convergence of a Class of Double-rank Minimization Algorithms 1. General Considerations. IMA J. Appl. Math. 1970, 6, 76-90.

40. Fletcher, R., A new approach to variable metric algorithms. Comput. J. 1970, 13, 317-322. 41. Goldfarb, D., A family of variable-metric methods derived by variational means. Math. Comput. 1970, 24, 23-26.

42. Shanno, D. F., Conditioning of quasi-Newton methods for function minimization. Math. Comput. 1970, 24, 647-656.

43. Gillespie, R. J., The electron-pair repulsion model for molecular geometry. J. Chem. Ed. 1970, 47, 18-23.

44. Khatib, F.; DiMaio, F.; Group, F. C.; Group, F. V. C.; Cooper, S.; Kazmierczyk, M.; Gildki, M.; Kryzywda, S.; Zabranska, H.; Pichova, I.; Thompson, J.; Popovic, Z.; Jaskolski, M.; Baker, D., Crystal structure of a monomeric retroviral protease solved by protein folding game players. Nat. Struct. Mol. Biol. 2011, 18, 1175-1177.

45. Khatib, F.; Desfosses, A.; Players, F.; Koepnick, B.; Flatten, J.; Popovic, Z.; Baker, D.; Cooper, S.; Gutsche, I.; Horowitz, S., Building de novo cryo-electron microscopy structure collaboratively with citizen scientists. PLoS Biol. 2019, 17, e3000472. 


\section{Section S1. Curriculum Exercises}

The following sections provided additional discussion for each exercise, including the goals for students, talking points for instructors, and typical outcomes.

\section{Section S1.1. Distorting Tetrahedral Methane}

This first exercise is intended to give students the time to familiarize themselves with both virtual reality (VR) and interactive molecular dynamics (IMD) simulations, while providing an opportunity for the instructor to gauge the students' level of chemical knowledge. The goal is for students to drag all four hydrogens into a planar conformation, typically by freezing two hydrogens in-plane with the carbon and then attempting to pull the final hydrogens into position. Instructors can discuss the 3D nature of molecular structure, ask about Valence Shell Electron Pair Repulsion (VSEPR) theory or orbital hybridization, and introduce the potential energy graphs as a quantitative measure of molecular stability (or lack thereof, in the case of planar methane). Students typically only see a planar methane for a split second, reinforcing the stability of tetrahedral methane.

\section{Section S1.2. Umbrella Flip in Ammonia}

The umbrella flip in ammonia is designed to introduce some of the limitations of chemical models the students may be familiar with. The goal is to invert the hydrogens in ammonia without simply rotating the molecule, a motion that is typically not possible with physical molecular modeling kits. Further discussion may build upon VSEPR and hybridization from Section S1.1, but with the introduction of lone pairs. Since applying only two forces in

ammonia necessarily applies a torque, students often rotate ammonia several times before a successful inversion event. As an additional challenge, the instructor can impose the restriction that only the nitrogen atom can be touched (usually also turning up the force multiplier to $4 \mathrm{x}$ as a concession). This secondary exercise is also typically completed by building up momentum in the symmetric bend vibrational mode, providing an opportunity for instructors to talk about normal modes. 


\section{Section S1.3. Psuedorotation of $\mathrm{PF}_{5}$ and $\mathrm{PH}_{3} \mathrm{~F}_{2}$}

The goal of this exercise is to introduce students to basic inorganic chemistry concepts such as hypervalency and pseudorotations of trigonal bipyramidal complexes. During our three outreach sessions, this section was located later in the curriculum and significantly less time was spent discussing these concepts as we often ran out of time. This is the best chance for an instructor to launch discussions surrounding inorganic chemistry, or heavier elements such as transition metals, as the other exercises feature solely organic molecules.

\section{Section S1.4. Ethane C-C Torsion}

The students are tasked with performing a torsional scan around the carbon-carbon single bond in ethane, which is fairly easily completed. If students have difficulty twisting the two halves of the molecule independently, one half can be frozen to help prevent the torsion of the entire molecule. Instructors can focus on the potential energy graph during the scan, as well as introduce organic chemistry concepts such as eclipsed vs. staggered conformers and gauche interactions.

\section{Section S1.5. Ethene $C=C$ Torsion}

This is a partner exercise to the ethane torsion in Section S1.4; this time, students are attempting to perform a torsional scan around the carbon-carbon double bond in ethene. Unlike the previous exercise, students are not typically successful with this torsion. Typical outcomes either involve a temporary success followed by the ethene untwisting itself, or a different mechanism where one hydrogen is almost completely removed from the ethene and then reattached to the other side. Instructors can turn on orbital isosurface rendering for the highest occupied molecular orbital (HOMO) to discuss the difference between sigma and pi bonds as an explanation for the difference between the two exercises.

\section{Section S1.6. Ring opening of cyclobutene}

In the first reactive exercise, students are tasked with performing a ring-opening reaction in cyclobutene. As with the ethane torsion in Section S1.4, students usually have no problems performing this task, but the exercise serves as a great launching pad for discussion. The HOMO can be visualized and there are several key differences when comparing to the orbitals seen in 
Section 1.5 with the ethene pi bond. During the course of the reaction, the molecular orbitals fluctuate greatly and may not always correspond to chemically intuitive ideas of orbitals. For more advanced students, this can lead to discussions of the properties and origins of molecular orbitals and caution against using blindly orbitals as a quantitative tool. The overall trend of evolution from carbon-carbon sigma bond, to carbon $p$ orbitals, to the formation of the new conjugated pi bond network depicted in Figure 5 can also serve as a lead-in to orbital symmetry rules (i.e. the Woodward-Hoffman rules) and mechanochemical concepts such as conrotatory vs disrotatory pathways.

\section{Section S1.7. Hydrogen atom through benzene}

The goal of this exercise is for students to push a hydrogen atom through the middle of a benzene ring. The starting geometry has been set such that with a significant amount of force, students can often achieve this on their first try; however, they will have much more difficulty on subsequent attempt as it is harder to achieve the proper alignment. Typically, these subsequent attempts will result in either complete repulsion or the occasional attachment/detachment of the hydrogen to the ring rather than pass through the center of the ring. Instructors can visualize the pi cloud above and below the benzene ring, showcasing how the apparent hole is not as large due to the significant electron density. As an aside, benzene also serves as an excellent reference for discussing aromaticity. The non-traditional nature of this exercise means that most students will not have a preexisting intuition for the outcome, making it an interesting testbed for learning new phenomena in VR-IMD simulations (compared to molecular modeling kits and Kekule-Lewis structures, which do not have much predictive power in this case).

\section{Section S1.8. Cyclohexane chair/boat conformer flip}

This exercise tasks the students with performing a chair-to-boat conformation flip in cyclobutene. There are typically two major blocking points for students, usually depending on whether they have seen this system before. Students who have never been seen the two structures may have some understanding the two conformers. Molecular modeling kits are extremely helpful in giving reference structures for those not in the VR simulation. The second issue is actually performing the conformer flip. Students usually only attempt to force the flip by pulling only on the carbons, which only works with an increased force modifier. A more successful 
strategy is to alternate pulling on the carbons and also adjusting the positions of the hydrogens in the half of the cyclohexane that is flipping up. As an instructor, providing these suggestions to the students leads naturally into discussion about equatorial vs. axial hydrogens and steric interactions.

\section{Section S1.9. Proton transfer in carbonic acid + water}

This is the first exercise designed for a more open-ended reactive experience. Students are placed in a simulation with a carbonic acid $\left(\mathrm{H}_{2} \mathrm{CO}_{3}\right)$ and four water molecules and tasked with transferring a proton from one molecule to another. Students can easily try multiple mechanisms but the most successful is pulling one proton from the carbonic acid onto a water. After several seconds, the proton will either hop back directly or a small proton relay will occur, typically ending with transfer back to the carbonic acid. Instructors can use this to discuss acidbase reactions, equilibrium constants, and proton wires (and also allude to the biochemical implications). One can also mention the role of solvent in tautomerization reactions, as these are usually drawn as the same hydrogen when that is often not the case.

\section{Section S1.10. $S_{N} 2$ of methyl fluoride + hydroxide}

The goal is for students to carry out the prototypical organic reaction of $\mathrm{S}_{\mathrm{N}} 2$ nucleophilic substitution. As with the hydrogen through benzene exercise in Section S1.7, the initial arrangement of the molecules makes it simple to succeed with the correct starting momentum, but difficult to realign for subsequent reactions. The alignment is mostly complicated by hydrogen bonding between the hydroxide and methyl group (for the forward reaction) and the fluoride ion and hydroxy group (for the reverse reaction). Given the strong connections to the standard organic chemistry curriculum, instructors can discuss the strength of nucleophiles and leaving groups while comparing the forward and reverse reactions, or the hydrogen inversion and reaction mechanism in general.

\section{Section S1.11. E1 of t-butyl fluoride + hydroxide}

This is a partner exercise to the $\mathrm{S}_{\mathrm{N}} 2$ reaction in Section $\mathrm{S} 1.10$, where instead the system uses $t$-butyl fluoride instead of methyl fluoride. This is nominally listed as the E1 reaction, but in 
reality both $\mathrm{S}_{\mathrm{N}} 1$ and $\mathrm{E} 1$ reactions occur frequently while the $\mathrm{S}_{\mathrm{N}} 2$ and $\mathrm{E} 2$ reactions can be triggered by a careful user. As with Section S1.7 and Section S1.10, the initial alignment favors the $S_{N} 1$ reaction if the student is quick to apply force to the fluoride leaving group and hydroxide nucleophile; otherwise, students typically perform the E1 reaction first as the alignment for the substitution reaction quickly degrades. This exercise is an excellent starting point for discussion about reaction competition and selectivity, as well as comparing the sterics of the $t$-butyl and methyl groups.

\section{Section S1.12. High Force Decomposition}

In our experience, students always become excited when they realize that applying high forces to completely destroy the molecule is an option. We've found the $t$-butyl system from Section S1.11 to be the best candidate system since it is the last exercise in the planned curriculum and also features the richest recombination products. It is also worth mentioning that high forces in the ethene torsion of Section S1.5 can sometimes see the formation of acetylene and hydrogen gas, which can also contribute to discussions about the relative strengths of bonds (i.e. it is easier to break carbon-hydrogen single bonds than it is to break a carbon-carbon double bond). 


\section{VR Instruction}

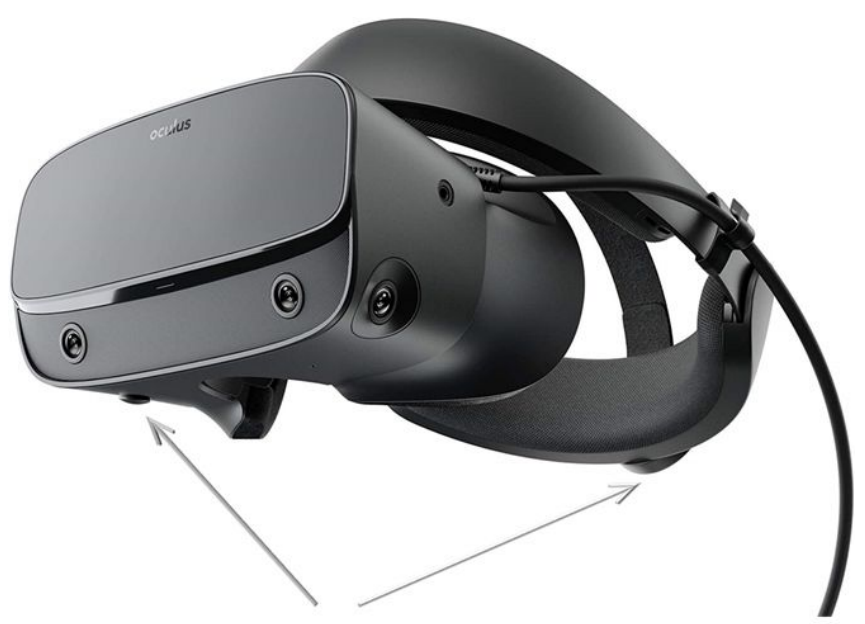

Adjust the size.

Menu button:

(All the same)

- Pause \& enter menu

- Resume \& leave menu

Binding: Teleport

Oculus Menu button:

(No function in this app)

- Enter or exit oculus menu

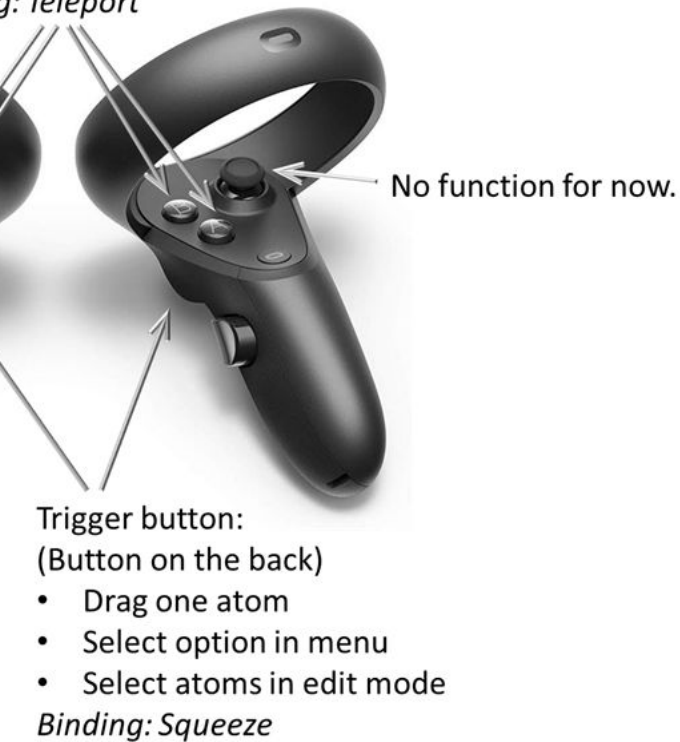




\section{Hardware requirements:}

GPU: $\quad$ NVIDIA GTX 960 / 1050Ti or greater

CPU: $\quad$ Intel i3-6100 or greater

Memory: $\quad 8 \mathrm{~GB}+\mathrm{RAM}$

VR: $\quad$ Oculus Rift S (or other VR devices)

Ports: $\quad$ DisplayPort 1.2 / Mini DisplayPort +1 x USB 3.0 (for Oculus Rift S)

\section{Software requirements:}

System: Windows 10

Driver: $\quad$ NVIDIA driver released after 2019

Oculus App (for Oculus Rift S)

SteamVR: $\quad$ Steam + SteamVR

Note: Oculus Rift S is the recommended VR device, because it's used in development, and a binding is provided. For other VR devices, you need to make your own customized binding. The names of the actions for binding is provided in the controller instruction figure.

\section{Instruction:}

1. General:

Press the menu button, hit the "Molecule" using the trigger button, in the "Molecule" dropdown list select the one you are interested. Exit the menu by hit the menu button again.

Reach your controller INTO one atom, and drag it using the trigger button. You can use two controllers to drag two atoms simultaneously.

Move the whole system using the grab button. Rotate the whole system by holding both grab buttons.

Discover other functions yourself!

2. Add a new molecule:

Generate an xyz file of your molecule or system. It is recommended that your system contains less than 20 atoms.

Open "VROption.txt", under the line "\#ifdef Molecule", add the complete absolute path of you xyz file. It will occur in the "Molecule" dropdown list.

If your molecule or system has a net charge ( -1 for example), in the second line of your $x y z$ file, which is intended for a description, write "charge $=-1.0$ ".

Note: Current backend is xtb 6.3 (semiempirical, https://github.com/grimme-lab/xtb). The TeraChem backend (HF) wil come soon. 


\section{Structure and Steric Effects}

Chemists like to use Lewis structures to show a simple picture of how atoms are bonded to one another in a molecule. Below are Lewis structures for methane, the simplest hydrocarbon, and ammonia, the simplest amine.<smiles>C</smiles>

Using your molecular modeling kit, build a methane and ammonia molecule and use them to answer the following questions.

1. What is the name of each shape (or how would you describe them)?

2. Using other atom pieces instead of the 4-hole black carbon, can you make any other geometrical shapes with four hydrogen atoms? Compared to the usual structure of methane, what makes your structure better or worse?

\section{VR Challenge 1: Planar Methane}

- Open the menu using the $A, B, X$, or $Y$ buttons. Point the controller to hover over buttons and click the index trigger to select them.

- Go to Molecule and select ch4 to open methane.

- Go to Dynamics > Force and select $\mathbf{4 x}$ to increase the force.

$\circ \quad$ Go to Edit > Freeze and select 2 hydrogen atoms and the carbon atom.

- Exit out of the menu by pressing the $A, B, X$, or $Y$ buttons again.

- Get the molecule in front of you by pressing and holding the middle finger grip trigger to move the entire scene - one hand pans, two hands rotate.

- Place your controllers over the non-frozen hydrogen atoms. Press and hold the index triggers to grab the atoms, and try to pull the atoms so that the molecule is flat. How easy is this to do? Is this structure stable?

- Pay attention to the potential energy diagram in the bottom left corner. Just like a ball rolling down a hill, the molecule is "more stable" when it has low potential energy. Does the graph support your previous answer or not? 
3. Looking just at the hydrogen atoms, one may have expected ammonia to be planar (i.e. all atoms in a single plane). Why do you think this is not the case?

4. Comparing the bond angles in methane with those in ammonia, which are wider? Why would this be the case?

\section{VR Challenge 2: Ammonia "Umbrella Flip"}

Consider the following two structures for ammonia, which are related by a 180 degree rotation. Convert your ammonia from hydrogen atoms down to hydrogen atoms up WITHOUT any rotation.

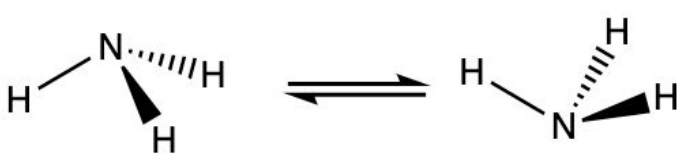

- Open the menu, make the following changes, and exit as above.

$\circ$ Go to Molecule and select $\mathbf{n h} 3$ to open ammonia.

- WITHOUT rotating the entire system, flip the hydrogen atoms from one side of the nitrogen to the other. How easy is this "umbrella flip" motion?

- For an extra challenge, see if you can do the flip while only touching the nitrogen atom.

$\circ$ If needed, go to Dynamics > Force and select $\mathbf{4 x}$ to increase the force.

\section{Single vs Double Bonds}

Use your molecular modeling kits to make ethane and ethene, shown below with a 3D representation of a Lewis diagram.<smiles>CC</smiles>

Ethane

$\left(\mathrm{C}_{2} \mathrm{H}_{6}\right)$<smiles>C=C</smiles>

Ethene

$\left(\mathrm{C}_{2} \mathrm{H}_{4}\right)$ 
5. List at least two geometric differences between ethane and ethene.

6. Is the $\mathrm{C}-\mathrm{C}$ bond in ethane or the $\mathrm{C}=\mathrm{C}$ bond in ethene easier to twist? Why do you think this is the case?

\section{VR Challenge 3: C-C Bond Torsions}

- Open the Molecule menu and select ethane.

- Spin the two methyl $\left(\mathrm{CH}_{3}\right)$ groups in opposite directions by pulling on the hydrogens.

- Now, open the Molecule menu and select ethene.

- Try to spin the two methylene $\left(\mathrm{CH}_{2}\right)$ groups in opposite directions as you did in ethane. Is this bond easier or harder to twist around? Approximately how much less or more force did you have to use?

- If more force is needed, open the menu and change Dynamics > Force to $\mathbf{4 x}$.

\section{Cyclic Molecules}

Molecules can also form cycles, such as cyclohexane (below left) and benzene (below right). Analogous to ethane and ethene above, cyclohexane is not flat while benzene is.
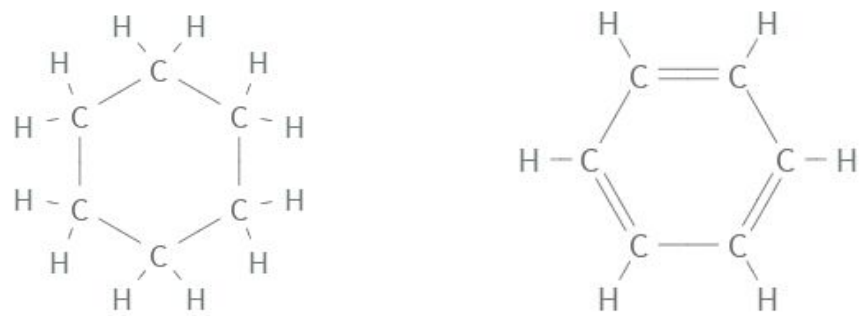

7. Cyclohexane has two main conformations: "chair" and "boat". Based on your molecular modeling kit, which conformer do you think is more stable and why?

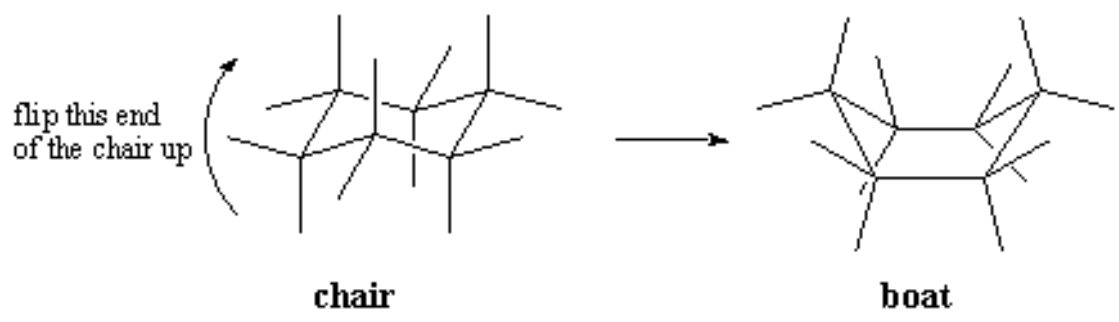




\section{VR Challenge 4: Cyclohexane Conformer Flip}

- Open the Molecule menu and select cyclohexane.

- Pull on atoms to switch between the "chair" and "boat". Do you notice any differences between what you expect to happen from the modeling kits and what actually happens?

8. Benzene is planar and only has one conformer for the nuclei. However, some of the electrons in benzene can change between configurations, an effect often called "resonance". Draw the two Lewis structures for benzene (often called "resonance structures").

\section{VR Challenge 5: Through the Benzene}

- Open the Molecule menu and select benzene+h.

- Try to pull the hydrogen atom through the benzene ring. Do you notice any differences between what is expected based on the modeling kits and the simulation?

$\circ$ If more force is needed, open the menu and change Dynamics > Force to $\mathbf{4 x}$.

\section{Hypervalency and Pseudorotations}

Molecules involving heavier atoms can take on interesting geometries as atoms can become hypervalent - that is, they can accept more than four bonds. This can lead to cool conformer rearrangements; for example, $\mathrm{PF}_{5}$ can perform a pseudorotation where the fluorine atoms can take a different configuration around the central phosphorus atom.

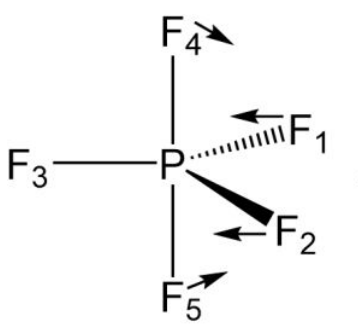

trigonal bipyramidal

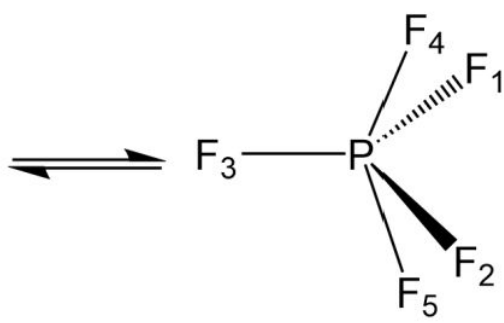

square pyramidal

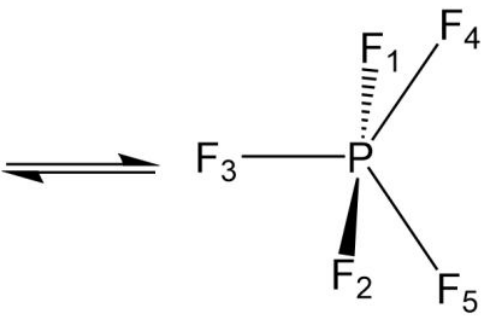

trigonal bipyramidal 
9. Consider the complex $\mathrm{PH}_{3} \mathrm{~F}_{2}$. Draw out all the different trigonal bipyramidal geometries can be formed with these atoms.

\section{VR Challenge 6: Pseudorotations}

- Open the Molecule menu and select ph3f2.

- Try to convert between the conformers of $\mathrm{PH}_{3} \mathrm{~F}_{2}$ without breaking any bonds. Did you find all the conformers and rotations between them with the modeling kit?

\section{Acid-Base Reactions}

Chemistry is most interesting when molecules react! You may have already heard of acid-base reactions, where an $\mathrm{H}^{+}$is transferred between two molecules.

10. Given carbonic acid $\left(\mathrm{H}_{2} \mathrm{CO}_{3}\right)$ and water $\left(\mathrm{H}_{2} \mathrm{O}\right)$, draw Lewis structures for the reactants and the most likely products. Using your molecular modeling kit, propose a mechanism for this reaction.

\section{VR Challenge 7: Not So Basic After All}

- Open the Molecule menu and select H2CO3_H2O.

- Try to carry out the acid-base reaction you proposed in Question 10. Is the system more stable (i.e. lower in energy) before or after the reactions? Why do you think this might be the case?

- If more force is needed, open the menu and change Dynamics $>$ Force to $4 \mathbf{x}$.

- As an added challenge, see if you can create a proton shuttle by making one $\mathrm{H}^{+}$transfer to water, transfer another $\mathrm{H}^{+}$between water molecules, and finally a third $\mathrm{H}^{+}$back to a different oxygen atom on the carbonic acid. 


\section{Simple Organic Reactions}

Chemical reactions that involve bond formation between heavier atoms (carbon, nitrogen, or oxygen) are very useful, as they can be used to build large molecules, such as pharmaceuticals for medicine, amino acids for proteins, or polymers for materials.

11. Consider the following substitution reaction between methyl fluoride and hydroxide:

$$
\mathrm{CH}_{3} \mathrm{~F}+\mathrm{OH}^{-} \Leftrightarrow \mathrm{CH}_{3} \mathrm{OH}+\mathrm{F}^{-}
$$

Using your molecular modeling kit, propose a mechanism for this reaction.

\section{VR Challenge 8: Substitution Reaction Part I}

- Open the Molecule menu and select MeF_OH.

- Try to carry out the substitution reaction you proposed in Question 11. Are there any other mechanisms that seem to work? Are there any competing reactions that lead to other products?

○ Open the Molecule menu, switch to any molecule, and switch back to MeF_OH to reset the system as needed.

12. Now consider a related substitution reaction with tert-butyl fluoride instead:

$$
\mathrm{C}\left(\mathrm{CH}_{3}\right)_{3} \mathrm{~F}+\mathrm{OH}^{-} \Leftrightarrow \mathrm{C}\left(\mathrm{CH}_{3}\right)_{3} \mathrm{OH}+\mathrm{F}^{-}
$$

Do you expect the most likely mechanism to change in any way?

\section{VR Challenge 9: Substitution Reaction Part II}

- Open the Molecule menu and select tBuF_OH.

- Try to carry out the substitution reaction with the new system. Does the system behave as you expect?

○ Open the Molecule menu, switch to any molecule, and switch back to tBuF_OH to reset the system as needed. 